\title{
Psychometric Properties of the Persian Generalized Trust Scale: Confirmatory Factor Analysis and Rasch Models and Relationship with Quality of Life, Happiness, and Depression
}

\author{
Chung-Ying $\operatorname{Lin}^{1}$ (D) - Vida Imani ${ }^{2} \cdot$ Mark D. Griffiths $^{3}$ (D) $\cdot$ Amir H. Pakpour $^{4,5}$ (D)
}

Published online: 20 May 2020

(C) Springer Science+Business Media, LLC, part of Springer Nature 2020

\begin{abstract}
The psychometric properties of the Generalized Trust Scale (GTS) are well established. Furthermore, previous studies have found that the GTS is positively associated with better mental health and lower distress, and the literature finds that trust is good for mental health. However, current literature does not have any psychometric evidence concerning the Persian GTS. This study translated the GTS into Persian and validated its psychometric properties. After translating the GTS into Persian using robust and standardized translation procedure, 1200 Iranians (mean age $=34.83$ years; 583 [48.6\%] males) completed the GTS, along with the Hospital Anxiety and Depression Scale (HADS), Short Form-12 (SF-12), and Oxford Happiness Questionnaire Short Form (OHQ-SF). The factor structure of Persian GTS was confirmed by a unidimensional model with a method factor (comparative fit index $=0.998$; Tucker-Lewis index $=0.992$ ). The unidimensional model was also supported by Rasch analysis (mean square $=0.75$ to 1.31 ). Other properties of the Persian GTS were satisfactory. More specifically, test-retest reliability was good (intraclass correlational coefficient $=0.865$ ), internal consistency was good $(\alpha=0.881$ ), and concurrent validity was supported (standardized $\beta=-0.086$ with depression in the HADS $[p=0.045] ;=-0.162$ with anxiety in the $\operatorname{HADS}[p<0.001]$; = 0.077 with mental component score in the SF-12 $[p=0.044] ;=0.624$ with OHQ-SF $[p<0.001])$. The six-item Persian GTS has promising psychometric properties and can be an effective measure to assess trust among Iranians.
\end{abstract}

Keywords Generalized Trust Scale $\cdot$ Persian validation · Psychometrics $\cdot$ Depression $\cdot$ Happiness
Abbreviations
CFI Confirmatory factor analysis
RMSEA Root mean square error of approximation
SRMR Standardized root mean square residual

Amir H. Pakpour

Pakpour_Amir@yahoo.com; apakpour@qums.ac.ir

Extended author information available on the last page of the article 


$\begin{array}{ll}\text { SEM } & \text { Structural equation model } \\ \text { TLI } & \text { Tucker-Lewis index } \\ \text { infit } & \text { Information-weighted fit statistic } \\ \text { MnSq } & \text { Mean square } \\ \text { outfit } & \text { Outlier-sensitive fit statistic }\end{array}$

Generalized trust (more commonly called general trust or trust) has been considered as one of the most important societal factors in maintaining an individual's daily living (Lundmark et al. 2016; Yamaguchi et al. 2014). More specifically, social interactions occurring in everyday life rely on trust having satisfactory social functioning at both micro and macro levels. Furthermore, it appears to be impossible to sustain good relationships or conduct business transactions without trust, and is arguably a type of social lubricant (Jasielska et al. 2019). Given that trust indicates the anticipation of positive (rather than negative) outcomes from others' actions (Ashraf et al. 2006; Johnson and Mislin 2011; Yamagishi and Yamagishi 1994), a positive relationship can be built up when two individuals involved in a social interaction both have trust. Furthermore, there are benefits for mental health among individuals who have higher levels of trust. For instance, substantial evidence from the literature indicates that a higher level of trust is associated with better mental health and lower level of distress (Abbott and Freeth 2008; Fahmi et al. 2019; Guo et al. 2018; Sato et al. 2018). Also, the literature suggests that people who have trust towards others are happier than those without trust (Tokuda et al. 2010).

A substantial body of empirical evidence shows that trust is associated with favorable national outcomes (e.g., economic growth and earnings) and various desirable interpersonal qualities, including volunteerism, donation to charity, social solidarity, tolerance, cooperation, and optimism (Ashraf et al. 2006; Rothstein and Uslaner 2005; Tov and Diener 2008). More specifically, studies using large samples have demonstrated a strong relationship between trust and the quality of agreeableness (Digman 1990; Evans and Revelle 2008). Moreover, a recently developed new Big Five Inventory incorporated the agreeableness facets (Soto and John 2017), and which indirectly demonstrated the importance of trust in the modern era. In brief, trust is an important societal factor because it associates with well being at both micro (individual) and macro (national) levels (Helliwell et al. 2016; Jasielska 2018; Tov and Diener 2008).

Given that previous research has highlighted the importance of trust (Helliwell et al. 2016; Jasielska 2018; Tov and Diener 2008), it is also important to accurately and precisely assess trust. At present, trust games and self-reports are the most commonly used methods to assess trust (Jasielska 2018). In a social context, trust games assess the latent construct of trust by examining how individuals make decisions to allocate money between themselves and strangers (Johnson and Mislin 2011). Unfortunately, interpreting such behavior in trust games is challenging and hard because the resulting behaviors can be influenced by different types of motivations and concepts, including risk-taking, social desirability, and/or betrayal aversion (Ashraf et al. 2006; Bohnet and Zeckhauser 2004; Butler et al. 2016).

In order to overcome the limitations of trust games, researchers can also use validated psychometric instruments to assess trust. The most extensively studied self-reported instruments on trust are the Interpersonal Trust Scale (ITS; Rotter 1967), World Values Survey (WVS; World Values Survey Association 2009), and Generalized Trust Scale (GTS; Yamagishi and Yamagishi 1994). The ITS comprises 25 items and was the first selfreported instrument developed assessing trust. The scale assesses trust in general and towards concrete social entities (e.g., parents and public officials). However, the scale has been 
criticized for its limited predictive validity (Carter and Mark Weber 2010; Evans and Revelle 2008). The WVS has a single item that asks whether individuals are generally trustworthy. However, the validity of this single item has been questioned due to the lack of clarity in whether it assesses trust or simply reflects the willingness of society to engage in trustworthy behaviors (Delhey et al. 2011; Glaeser et al. 2000). The GTS is an alternative option to the aforementioned two instruments. Furthermore, the GTS has satisfactory features in predictive validity (i.e., successfully predicts trusting behavior) and been validated across cultures (Carter and Mark Weber 2010; Montoro et al. 2014; Yamaguchi et al. 2014).

Although the GTS has good psychometric properties, to the best of the present study's authors' knowledge, it has never been validated in an Iranian context. Therefore, the present study translated the GTS into a culturally appropriate Persian version with adequate procedure that ensured the linguistic validity of the Persian GTS. Moreover, the psychometric properties of Persian GTS were evaluated among a relatively large sample of Iranian adults to better understand the extent to which the Persian GTS can be applied. More specifically, three types of psychometric testing were utilized: (i) classical test theory to understand the factor structure, item properties, reliability, and reproducibility (i.e., test-retest reliability) of the Persian GTS; (ii) Rasch analysis to reexamine the factor structure and item properties of the Persian GTS and to further examine the separation reliability and differential functioning item (DIF) of the Persian GTS; and (iii) external criteria (depression, anxiety, physical and mental components in the quality of life, and happiness) to examine the concurrent validity of the Persian GTS through constructed regression models, of which demographics of age and gender are controlled for.

\section{Methods}

\section{Participants and Procedure}

The present study was conducted to participants who were household residents in the Qazvin province between December 2018 and July 2019. The study sample was selected using a random systematic and cluster sampling with help of postcodes. The 10-digit postcode and their related addresses were drawn from the databank registry of Qazvin central post office. Three trained research assistants approached the selected houses and conducted door-to-door interviews face-to-face. The study population was residents 18 years of age and older and who agreed to participate. The study protocol was approved by the Ethics Committee of Qazvin University of Medical Sciences, and all participants $(n=1200)$ provided written informed consent (IR.QUMS.REC.1397.122).

\section{Measures}

\section{Generalized Trust Scale}

The GTS comprises six items rated on a five-point Likert scale $(1=$ completely disagree; $5=$ completely agree), and assesses an individual's general level of trust (Yamagishi and Yamagishi 1994; Yamagishi and Kosugi 1999). A higher score on the GTS indicates a higher level of trust. Previous findings have demonstrated the good psychometric properties for the GTS (Jasielska et al. 2019). A sample item of the GTS is "Most people are basically honest." 
The Persian GTS was translated using the standard method proposed by international guidelines (Beaton et al. 2000; Pakpour et al. 2014). More specifically, the English GTS was first translated into Persian by two independent translators who were not aware of the GTS prior to translation. Then, the two independent forward translations were reconciled into one version, which was used for back translation. The back translation was done by another two independent translators who were not involved in the forward translation and reconciliation. A panel including different disciplines compared all the GTS versions (two forward translations, two back translations, and the English GTS) and agreed an interim version of the Persian GTS. The interim version was pilot tested and underwent cognitive briefing to ensure its readability before it was used for formal psychometric testing in the present study.

\section{Short Form-12}

The Short Form-12 (SF-12) comprises 12 items rated on either a Likert scale (from three-point to six-point scale) or a dichotomous scale (yes and no) and assesses an individual's quality of life (Hagell and Westergren 2011). The 12 items were used to attain a physical component score and a mental component score, where a higher score of each component indicates a higher level of quality of life. Previous findings have demonstrated the good psychometric properties for the Persian SF-12 (Pakpour et al. 2011). A sample item of the SF-12 physical component score is "Does your health now limit you from climbing several flights of stairs?" and a sample item of the SF-12 mental component score is "Have you felt downhearted and blue?"

\section{Oxford Happiness Questionnaire Short Form}

The Oxford Happiness Questionnaire Short Form (OHQ-SF) comprises eight items rated on a six-point Likert scale ( $1=$ strongly disagree; $6=$ strongly agree $)$ and assesses an individual's happiness (Hills and Argyle 2002). A higher score indicates a higher level of depression or anxiety. Previous findings have demonstrated the good psychometric properties for the Persian OHQ-SF (Alipour and Agah Heris 2007). A sample item of the OHQ-SF is "I feel that life is very rewarding."

\section{The Hospital Anxiety and Depression Scale}

The Hospital Anxiety and Depression Scale (HADS) comprises 14 items rated on a four-point Likert scale (score from 0 to 3), and assesses an individual's depression (seven items) and anxiety (seven items) (Montazeri et al. 2003). A higher score indicates a higher level of depression or anxiety. Previous findings have demonstrated the good psychometric properties for the Persian HADS (Lin and Pakpour 2017). A sample item of the HADS depression subscale is "I feel as if I am slowed down" and a sample item of the HADS anxiety subscale is "I feel tense or wound up."

\section{Data Analysis}

The socio-demographics of the participants were analyzed using descriptive statistics. The psychometric properties of the Persian GTS were then analyzed. Ceiling and floor effects were calculated with an acceptable value at $<20 \%$ (Lin et al. 2018a). Construct validity of the 
Persian GTS was assessed using the confirmatory factor analysis (CFA) using the diagonally weighted least squares (WLSMV) estimator. Moreover, two factor structures were proposed for the Persian GTS according to the prior research (Jasielska et al. 2019): a unidimensional model without method factor and a unidimensional model with method factor. The two models were compared using the $\chi^{2}$ difference test to determine which one performs better. In addition to the $\chi^{2}$ difference test, comparative fit index $(\mathrm{CFI})>0.9$, Tucker-Lewis index (TLI) $>0.9$, root mean square error of approximation $(\mathrm{RMSEA})<0.08$, and standardized root mean square residual $(\mathrm{SRMR})<0.08$ were used to understand whether the proposed model has supported factor structure (Fung et al. 2019; Lin 2018). Average variance extracted $>0.5$, composite reliability $>0.6$, and internal consistency of Cronbach's $\alpha>0.7$ were also used to support the acceptable psychometric properties of the Persian GTS. Furthermore, intraclass correlation coefficient (ICC) $>0.75$ indicates excellent test-retest reliability of the Persian GTS (Koo and Li 2016).

Rasch analysis utilizing a Rasch rating scale model (RSM) was used to further corroborate the psychometric properties of the Persian GTS. The Persian GTS items were tested using the information-weighted fit statistic (infit) mean square (MnSq) and the outlier-sensitive fit statistics (outfit) MnSq. Infit and outfit MnSq valued between 0.5 and 1.5 indicate the Persian GTS item fitted the trust construct (Lin et al. 2018b, c). The response scale of the Persian GTS was examined using the average and step measures of the difficulty in each response (i.e., each point of the five-point Likert scale). The average and step measures should be monotonically increased to indicate a good response ordering. Differential item functioning (DIF) of the Persian GTS was assessed using the DIF contrast across gender (male vs. female), place of residence (urban vs. rural residence), age (higher than a mean age of 34.8 years vs. lower than mean age at 34.8 years), number of years in education (higher than mean of 10.11 years vs. lower than mean of 10.11 years), and self-reported health (poor vs. good). A DIF contrast $<0.5$ indicates no substantial DIF across the subgrouping (Lin et al. 2018b, c).

Several regression models were constructed to test the concurrent validity of the Persian GTS. The regression models share the same independent variable of Persian GTS and controlled variables of age and gender. The dependent variables of the regression models were depression, anxiety, physical component and mental component scores, and happiness.

\section{Results}

The mean age of the 1200 participants was 34.83 years $(\mathrm{SD}=11.97)$. The mean educational years of the participants was $10.11(\mathrm{SD}=5.09)$. Nearly half of the participants were male $(n=$ $583 ; 48.6 \%)$ and most of the participants self-reported as being in good health $(n=981$; $81.7 \%)$. Other participants' characteristics are shown in Table 1.

The Persian GTS had low ceiling $(0.5 \%)$ and floor effects $(2.0 \%)$. Both proposed factor structures for the Persian GTS (i.e., the unidimensional structure with and without the method factor) had satisfactory fit indices in the CFA (Fig. 1). The factor structure with the method effect slightly and significantly outperformed the structure without the method effect $\left(\Delta \chi^{2}=\right.$ $5.905, d f=1 ; p=0.02$ ). Additionally, average variance extracted (0.562), composite reliability (0.883), and internal consistency of Cronbach's $\alpha$ (0.881) were good for the Persian GTS. Test-retest reliability of the Persian GTS in a two-week interval was also good $(\mathrm{ICC}=0.865)$.

Satisfactory fit of the Persian GTS was also shown in the Rasch model results. Item separation reliability (0.98) and person separation reliability (0.87) were high; item separation 
Table 1 Socio-demographic characteristics of participants

\begin{tabular}{lll}
\hline & Mean or $n$ & SD or \% \\
\hline Age (year) & 34.83 & 11.97 \\
Educational year & 10.11 & 5.09 \\
Gender & & 48.6 \\
Male & 583 & 51.4 \\
Female & 617 & 30.7 \\
Marital status & & 49.3 \\
Single & 368 & 20.0 \\
Married & 592 & 67.3 \\
Divorced & 240 & 36.3 \\
Having children & & \\
Yes & 765 & 40.2 \\
$\quad$ No & 435 & 59.8 \\
Place of residence & & \\
Urban & 482 & 49.3 \\
Rural & 718 & 16.8 \\
Occupational status & & 8.0 \\
Employed & 592 & 20.9 \\
Unemployed & 201 & 5.0 \\
Student & 96 & 81.7 \\
Housewife & 251 & 18.3 \\
Retired & 60 & \\
Self-rated health & 981 & \\
Good & 219 & \\
Poor & & \\
\hline
\end{tabular}

index (7.12) and person separation index from Rasch (2.62) were large. Moreover, both infit and outfit MnSq were valued between 0.5 and 1.5 for all the Persian GTS items (Table 2). The response of the five-point Likert scale in the Persian GTS increased its difficulty monotonically (average measure from -3.25 to 2.79 ; step measure from -3.82 to 3.80 ) (Table 3 ). Furthermore, no DIF items were displayed for the Persian GTS across gender, place of residence, age, number of years of education, and self-reported health (Table 4).

Concurrent validity of the Persian GTS was supported by the significant associations found in the regression models (Table 5). More specifically, the higher score on the Persian GTS was significantly associated with lower level of depression (standardized $\beta=-0.086 ; p=0.045$ ), lower level of anxiety (standardized $\beta=-0.023 ; p<0.001$ ), higher level of mental component score in SF-12 (standardized $\beta=0.077 ; p=0.044$ ), and higher level of happiness (standardized $\beta=0.624 ; p<0.001)$.

\section{Discussion}

No previous study has ever translated the GTS into Persian and examined its psychometric properties. Therefore, the present study findings extend the current psychometric evidence of the GTS from previously tested cultures (e.g., USA and Japan) (Carter and Mark Weber 2010; Montoro et al. 2014; Yamaguchi et al. 2014) to the Middle East of Iran. Moreover, the Persian GTS was translated robustly with methodological rigor (i.e., following the use of international guidelines with appropriate cultural adaption). In comparing the psychometric findings from present study with those from other countries (Carter and Mark Weber 2010; Jasielska et al. 


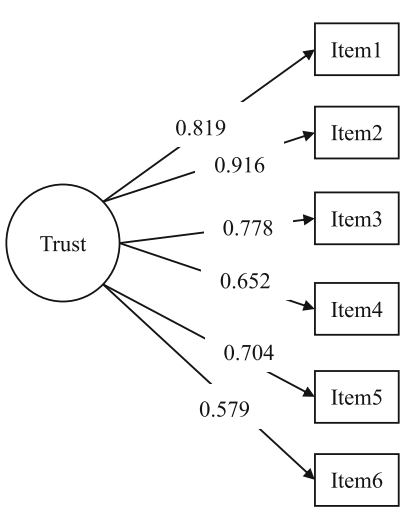

(a) Unidimensional model

Fit indices

$\chi^{2}(d f)=19.161(9)$

comparative fit index $=0.995$

Tucker-Lewis index $=0.986$

RMSEA $=0.053$

$90 \% \mathrm{CI}$ of $\mathrm{RMSEA}=0.001,0.098$

$\mathrm{SRMR}=0.018$

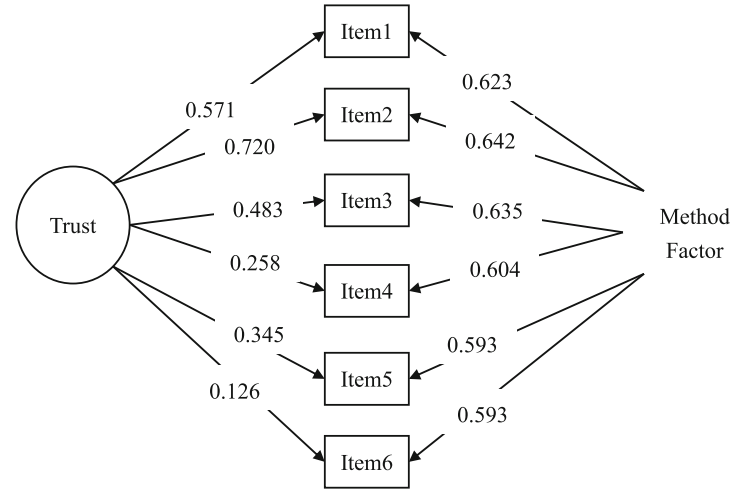

(b) Unidimensional model with method effect

Fit indices

$\chi^{2}(d f)=13.256(8)$

comparative fit index $=0.998$

Tucker-Lewis index $=0.992$

RMSEA $=0.041$

$90 \%$ CI of RMSEA $=0.001,0.093$

$\mathrm{SRMR}=0.014$

Fig. 1 Confirmatory factor analysis results of the Generalized Trust Scale. a Without method factor. b With method factor. RMSEA root mean square error of approximation, SRMR standardized root mean square residual, CI confidence interval

2019; Montoro et al. 2014; Yamaguchi et al. 2014), the GTS structure was confirmed as being a single factor (i.e., trust). Moreover, the present study's findings corroborate the proposed method factor proposed in Jasielska et al.'s (2019) recent study. Furthermore, the present study's findings concerning internal consistency and concurrent validity also confirm the findings of prior research (Carter and Mark Weber 2010; Montoro et al. 2014; Yamaguchi et al. 2014).

Apart from the psychometric evidence shown in the classical test theory (i.e., CFA, internal consistency, test-retest results), Rasch analysis results found in the present study further emphasized the validity of the Persian GTS. By applying different types of methods to demonstrate the promising psychometric properties, researchers can be confident in using the Persian GTS to assess trust levels among Iranians. More specifically, the Rasch results suggested the following. First, the Persian GTS items all fit in the trust construct with only little redundancy or misfit exists among these items because all the items had acceptable infit

Table 2 Rasch difficulties and fit statistics for each item of the Persian Generalized Trust Scale

\begin{tabular}{llllll}
\hline Item description & Difficulty & $\begin{array}{l}\text { Infit } \\
\mathrm{MnSq}\end{array}$ & $\begin{array}{l}\text { Outfit } \\
\mathrm{MnSq}\end{array}$ & $\begin{array}{l}\text { Model } \\
\mathrm{SE}\end{array}$ & Correlation \\
\hline 1. Most people are basically honest & 0.19 & 0.86 & 0.83 & 0.07 & 0.81 \\
2. Most people are trustworthy & 0.45 & 0.79 & 0.76 & 0.07 & 0.81 \\
3. Most people are basically good and kind & -0.55 & 0.83 & 0.75 & 0.08 & 0.82 \\
4. Most people are trustful of others & 0.01 & 1.12 & 1.13 & 0.07 & 0.73 \\
5. I am trustful & 0.77 & 0.96 & 0.95 & 0.08 & 0.75 \\
6. Most people will respond in kind when they are & -0.86 & 1.31 & 1.30 & 0.08 & 0.72 \\
trusted by others & & & & & \\
\hline
\end{tabular}

Infit information-weighted fit statistic, Outfit outlier-sensitive fit statistics, $M n S q$ mean square, $S E$ standard error 
Table 3 Response disordering tests on Persian Generalized Trust Scale

\begin{tabular}{lllll}
\hline & Average measure & Step measure & Infit MnSq & Outfit MnSq \\
\hline Score 1 & -3.25 & - & 1.19 & 1.08 \\
Score 2 & -1.38 & -3.82 & 0.82 & 0.84 \\
Score 3 & 0.16 & -0.05 & 0.88 & 0.86 \\
Score 4 & 1.30 & 0.07 & 0.92 & 0.94 \\
Score 5 & 2.79 & 3.80 & 1.56 & 1.26 \\
\hline
\end{tabular}

1: strongly disagree; 2: disagree; 3: neutral; 4: agree; 5: strongly agree

Infit information-weighted fit statistic, Outfit outlier-sensitive fit statistics, $M n S q$ mean square

and outfit MnSq from the Rasch model (i.e., ranged between 0.5 and 1.5) (Lin et al. 2018b, c). Second, all the Persian GTS items were interpreted similarly across subgroups (including gender, place of residence, age, number of years education, and self-rated health subgroups) because of the negligible DIF contrasts (i.e., DIF contrast < 0.5) (Lin et al. 2018b, c).

With the satisfactory psychometric properties shown for the Persian GTS, Iranian programs on increasing trust may use the Persian GTS to examine whether the program is effective. As negative consequences of a low trust level have been found among those with lowered subjective well being, decreased social capital, and impaired society's resilience (Helliwell et al. 2016; Tov and Diener 2008), rebuilding trust seems to be desirable. Prior research has suggested that social trust in groups and societies may be elevated by effective training and social campaigns that have integrated trust and kindness (Jasielska 2018). With learning to trust others through kindness practice, people may therefore elevate their happiness level and increase their willingness to engage in prosocial behaviors because social interactions must be derived from a foundation of trust (Jasielska et al. 2019). Moreover, such social connection and social support are key components that lead to satisfactory subjective well being (Wang 2016), resilience ability to cope with stress (Pietrzak et al. 2010), and a healthy and happy life

Table 4 Differential item functioning (DIF) across different subgroups in the Persian Generalized Trust Scale

\begin{tabular}{llcllr}
\hline Item no. & $\begin{array}{l}\text { DIF } \\
\text { contrast } \\
\text { across } \\
\text { gender }\end{array}$ & $\begin{array}{l}\text { DIF contrast } \\
\text { across place } \\
\text { of residence }\end{array}$ & $\begin{array}{l}\text { DIF } \\
\text { contrast } \\
\text { across } \\
\text { age }^{\mathrm{c}}\end{array}$ & $\begin{array}{l}\text { DIF } \\
\text { contrast } \\
\text { across } \\
\text { education }\end{array}$ & $\begin{array}{l}\text { d } \\
\text { across self- } \\
\text { rated health }\end{array}$ \\
\hline 1. Most people are basically honest & 0.01 & 0.19 & 0.06 & 0.23 & -0.03 \\
2. Most people are trustworthy & -0.24 & 0.18 & 0.18 & 0.14 & -0.11 \\
3. Most people are basically good and kind & 0.18 & -0.15 & -0.23 & -0.06 & 0.13 \\
4. Most people are trustful of others & -0.06 & 0.22 & 0.01 & -0.14 & 0.29 \\
5. I am trustful & 0.16 & -0.20 & -0.05 & 0.25 & -0.07 \\
6. Most people will respond in kind when & -0.03 & -0.03 & 0.02 & -0.08 & 0.05 \\
they are trusted by others & & & & & \\
\hline
\end{tabular}

DIF contrast $=$ difficulty in subgroup 1 - difficulty in subgroup 2, and all DIF contrasts were nonsignificant a Patients were classified into men (subgroup 1) and female (subgroup 2)

${ }^{b}$ Patients were classified into residency in urban areas (subgroup 1) and residency in rural areas (subgroup 2)

c Patients were classified into older (higher than mean age $>34.83$; subgroup 1) and younger (lower than mean age $\leq 34.83$; subgroup 2)

d Patients were classified into higher educated (higher than mean education years $>10.11$; subgroup 1) and lower educated (lower than mean education years $\leq 10.11$; subgroup 2)

e Patients were classified into poor self-reported health (subgroup 1) and good self-reported health (subgroup 2) 
Table 5 Concurrent validity of the Persian Generalized Trust Scale using regression models

\begin{tabular}{lcccc}
\hline Variable & $\beta(\mathrm{SE})$ & Stand. $\beta$ & $t(p$ value $)$ & $95 \%$ CI \\
\hline Depression & $-0.015(0.008)$ & -0.086 & $-2.01(0.045)$ & $-0.030,-0.003$ \\
Anxiety & $-0.023(0.006)$ & -0.162 & $-3.76(<0.001)$ & $-0.035,-0.011$ \\
Physical component score & $0.004(0.002)$ & 0.064 & $1.674(0.09)$ & $-0.001,0.009$ \\
Mental component score & $0.006(0.003)$ & 0.077 & $2.014(0.044)$ & $0.001,0.012$ \\
Happiness & $0.158(0.01)$ & 0.624 & $16.628(<0.001)$ & $0.139,0.177$ \\
\hline
\end{tabular}

All regression models have adjusted for age and gender

SE standard error, $C I$ confidence interval

Depression and anxiety were assessed using Hospital Anxiety and Depression Scale; physical component score and mental component score were assessed using Short Form 12 (SF-12); happiness was assessed using Oxford Happiness Questionnaire Short Form

(Holt-Lunstad et al. 2017). Therefore, these types of positive association may have potential ability in improving subjective well being for individuals and increasing social capital for the countries where they reside.

There are some limitations in the present study. First, the study sample was only Iranian and therefore it is questionable whether the Persian GTS also has strong psychometric properties in other Persian-speaking populations, such as those in Afghanistan. Therefore, in order to strengthen the generalizability of the Persian GTS, future studies are suggested to recruit Persian-speaking individuals living in different countries and retest the psychometric properties of the Persian GTS. Second, although the test-retest reliability was satisfactory in a 2-week interval, and which indicated the reproducibility of the Persian GTS, the responsiveness for the Persian GTS remains unclear. In other words, the present study's findings cannot support whether the Persian GTS can detect the change of trust level among a group who receives a trust intervention. Future studies are therefore warranted to investigate whether the Persian GTS has the feature of good responsiveness. Third, the external criteria used in the present study were self-reported by the participants. Given that Persian GTS is also self-report, common method bias might exist in the concurrent validity findings. That is, the associations found between the Persian GTS and other external criteria might be due to the same method in the assessment (i.e., self-report). Similarly, some biases resulting from self-reports such as social desirability and recall bias might also exist and jeopardize the present study's findings.

\section{Conclusion}

The present study demonstrated that the six-item Persian GTS is a simple measure to assess trust levels among Iranians. The psychometric properties of the Persian GTS were satisfactory among a large sample of Persian-speaking Iranians. Moreover, the psychometric properties were supported by rigorous analyses, including those conducted using classical test theory and those using Rasch models.

Acknowledgments The authors wish to thank the participants for generously giving their time and insight. 


\section{Compliance with Ethical Standards}

Conflict of Interest The authors declare that they have no conflict of interest.

Informed Consent Statement All procedures followed were in accordance with the ethical standards of the responsible committee on human experimentation (institutional and national) and with the Helsinki Declaration of 1975 , as revised in 2000. Informed consent was obtained from all patients for being included in the study.

\section{References}

Abbott, S., \& Freeth, D. (2008). Social capital and health: Starting to make sense of the role of generalized trust and reciprocity. Journal of Health Psychology, 13(7), 874-883.

Alipour, A., \& Agah Heris, M. (2007). Reliability and validity of the Oxford Happiness Inventory among Iranians. Journal of Iranian Psychologists, 3(12), 287-298.

Ashraf, N., Bohnet, I., \& Piankov, N. (2006). Decomposing trust and trustworthiness. Experimental Economics, 9, 193-208.

Beaton, D. E., Bombardier, C., Guillemin, F., \& Ferraz, M. B. (2000). Guidelines for the process of cross-cultural adaptation of self-report measures. Spine, 25, 3186-3191. https://doi.org/10.1097/00007632-20001215000014.

Bohnet, I., \& Zeckhauser, R. (2004). Trust, risk and betrayal. Journal of Economic Behavior and Organization, $55,467-484$.

Butler, J. V., Giuliano, P., \& Guiso, L. (2016). Trust and cheating. The Economic Journal, 126, 1703-1738.

Carter, N. L., \& Mark Weber, J. (2010). Not Pollyannas: Higher generalized trust predicts lie detection ability. Social Psychological and Personality Science, 1, 274-279.

Delhey, J., Newton, K., \& Welzel, C. (2011). How general is trust in "most people"? Solving the radius of trust problem. American Sociological Review, 76(5), 786-807.

Digman, J. M. (1990). Personality structure: Emergence of the five-factor model. Annual Review of Psychology, $41,417-440$.

Evans, A. M., \& Revelle, W. (2008). Survey and behavioral measurements of interpersonal trust. Journal of Research in Personality, 42, 1585-1593.

Fahmi, M., Panjaitan, N. A., Habibie, I., Siregar, A. Y. M., Amarullah, G., Rahma, S., \& D. K. (2019). Does your neighborhood protect you from being depressed? A study on social trust and depression in Indonesia. $B M C$ Public Health, 19(1), 1371.

Fung, X. C. C., Pakpour, A. H., Wu, K.-Y., Fan, C.-W., Lin, C.-Y., \& Tsang, H. H. W. (2019). Psychosocial variables related to weight-related self-stigma in physical activity among young adults across weight status. International Journal of Environmental Research and Public Health, 17, 64.

Glaeser, E. L., Laibson, D. I., Scheinkman, J. A., \& Soutter, C. L. (2000). Measuring trust. The Quarterly Journal of Economics, 115, 811-846.

Guo, C., Tomson, G., Keller, C., \& Söderqvist, F. (2018). Prevalence and correlates of positive mental health in Chinese adolescents. BMC Public Health, 18(1), 263.

Hagell, P., \& Westergren, A. (2011). Measurement properties of the SF-12 health survey in Parkinson's disease. Journal of Parkinson's Disease, 1(2), 185-196.

Helliwell, J., Huang, H., \& Wang, S. (2016). The distribution of the world happiness. In J. Helliwell, R. Layard, \& G. Sachs (Eds.), World happiness report 2016 (pp. 8-49). New York: Sustainable Development Solutions Network.

Hills, P., \& Argyle, M. (2002). The Oxford Happiness Questionnaire: A compact scale for the measurement of psychological well-being. Personality and Individual Differences, 33(7), 1073-1082. https://doi.org/10.1016 /S0191-8869(01)00213-6.

Holt-Lunstad, J., Robles, T. F., \& Sbarra, D. A. (2017). Advancing social connection as a public health priority in the United States. American Psychologist, 72, 517-530.

Jasielska, D. (2018). The moderating role of kindness on the relation between trust and happiness. Current Psychology, Advance online publication, 1-9. https://doi.org/10.1007/s12144-018-9886-7.

Jasielska, D., Rogoza, R., Zajenkowska, A., \& Russa, M. B. (2019). General trust scale: Validation in crosscultural settings. Current Psychology. https://doi.org/10.1007/s12144-019-00435-2.

Johnson, N. D., \& Mislin, A. A. (2011). Trust games: A meta-analysis. Journal of Economic Psychology, 32, $865-889$. 
Koo, T. K., \& Li, M. Y. (2016). A guideline of selecting and reporting intraclass correlation coefficients for reliability research. Journal of Chiropractic Medicine, 15(2), 155-163.

Lin, C.-Y. (2018). Comparing quality of life instruments: Sizing Them Up versus PedsQL and Kid-KINDL. Social Health \& Behavior, 1, 42-47.

Lin, C.-Y., \& Pakpour, A. H. (2017). Using Hospital Anxiety and Depression Scale (HADS) on patients with epilepsy: Confirmatory factor analysis and Rasch models. Seizure: European Journal of Epilepsy, 45, 4246.

Lin, C.-Y., Ganji, M., Pontes, H. M., Broström, A., Griffiths, M. D., \& Pakpour, A. H. (2018a). Psychometric evaluation of the Persian Internet Disorder Scale (IDS-15) among adolescents. Journal of Behavioral Addictions, 7(3), 665-675.

Lin, C.-Y., Ou, H.-T., Nikoobakht, M., Broström, A., Årestedt, K., \& Pakpour, A. H. (2018b). Validation of Medication Adherence Report Scale (MARS-5) in older stroke patients in Iran. Journal of Cardiovascular Nursing, 33(6), 536-543.

Lin, C.-Y., Pakpour, A. H., Broström, A., Fridlund, B., Årestedt, K., Strömberg, A., Jaarsma, T., \& Mårtensson, J. (2018c). Psychometric properties of the 9-item European Heart Failure Self-Care Behavior Scale using confirmatory factor analysis and Rasch analysis among Iranian patients. Journal of Cardiovascular Nursing, 33(3), 281-288.

Lundmark, S., Gilljam, M., \& Dahlberg, S. (2016). Measuring generalized trust: An examination of question wording and the number of scale points. Public Opinion Quarterly, 80(1), 26-43.

Montazeri, A., Vahdaninia, M., Ebrahimi, M., \& Jarvandi, S. (2003). The Hospital Anxiety and Depression Scale (HADS): Translation and validation study of the Iranian version. Health and Quality of Life Outcomes, 1, 14.

Montoro, A., Shih, P. C., Román, M., \& Martínez-Molina, A. (2014). Spanish adaptation of Yamagishi General Trust Scale. Anales de Psicología/Annals of Psychology, 30, 302-307.

Pakpour, A. H., Nourozi, S., Molsted, S., Harrison, A. P., Nourozi, K., \& Fridlund, B. (2011). Validity and reliability of Short Form-12 Questionnaire in Iranian hemodialysis patients. Iranian Journal of Kidney Diseases, 5(3), 175-181.

Pakpour, A. H., Zeidi, I. M., Yekaninejad, M. S., \& Burri, A. (2014). Validation of a translated and culturally adapted Iranian version of the International Index of Erectile Function. Journal of Sex and Marital Therapy, 40(6), 541-551.

Pietrzak, R. H., Johnson, D. C., Goldstein, M. B., Malley, J. C., Rivers, A. J., Morgan, C. A., \& Southwick, S. M. (2010). Psychosocial buffers of traumatic stress, depressive symptoms, and psychosocial difficulties in veterans of operations enduring freedom and Iraqi freedom: The role of resilience, unit support, and post deployment social support. Journal of Affective Disorders, 120, 188-192.

Rothstein, B., \& Uslaner, E. M. (2005). All for all: Equality, corruption, and social trust. World Politics, 58, 4172.

Rotter, J. B. (1967). A new scale for the measurement of interpersonal trust. Journal of Personality, 35(4), 651665.

Sato, Y., Aida, J., Tsuboya, T., Shirai, K., Koyama, S., Matsuyama, Y., Kondo, K., \& Osaka, K. (2018). Generalized and particularized trust for health between urban and rural residents in Japan: A cohort study from the JAGES project. Social Science and Medicine, 202, 43-53.

Soto, C. J., \& John, O. P. (2017). The next big five inventory (BFI-2): Developing and assessing a hierarchical model with 15 facets to enhance bandwidth, fidelity, and predictive power. Journal of Personality and Social Psychology, 113, 117-143.

Tokuda, Y., Fujii, S., \& Inoguchi, T. (2010). Individual and country-level effects of social trust on happiness: The Asia Barometer Study. Journal of Applied Social Psychology, 40(10), 2574-2593.

Tov, W., \& Diener, E. (2008). The well-being of nations: Linking together trust, cooperation, and democracy. In B. A. Sullivan, M. Snyder, \& J. L. Sullivan (Eds.), Cooperation: The political psychology of effective human interaction (pp. 323-342). Malden: Blackwell.

Wang, X. (2016). Subjective well-being associated with size of social network and social support of elderly. Journal of Health Psychology, 21, 1037-1042.

World Values Survey Association, ed. 2009. World Values Survey 2005 Official Data File (version 20090901 at www.worldvaluessurvey.org).

Yamagishi, T., \& Kosugi, M. (1999). Cheater detection in social exchange. Cognitive Studies, 6, 179-190.

Yamagishi, T., \& Yamagishi, M. (1994). Trust and commitment in the United States and Japan. Motivation and Emotion, 18, 129-166.

Yamaguchi, H., Brase, G. L., \& Yama, H. (2014). Two methods to measure the level of trust of Americans and Japanese: A cross-cultural study. Japanese: A cross-cultural study. SAGE Open, 1-7. https://doi.org/10.1177 $/ 2158244014556991$. 
Publisher's Note Springer Nature remains neutral with regard to jurisdictional claims in published maps and institutional affiliations.

\section{Affiliations}

\section{Chung-Ying Lin $^{1} \cdot$ Vida Imani ${ }^{2} \cdot$ Mark D. Griffiths $^{3} \cdot$ Amir H. Pakpour ${ }^{4,5}$}

1 Department of Rehabilitation Sciences, The Hong Kong Polytechnic University, Hung Hom, Hong Kong

2 Pediatric Health Research Center, Tabriz University of Medical Sciences, Tabriz, Iran

3 International Gaming Research Unit, Psychology Department, Nottingham Trent University, Nottingham, UK

4 Social Determinants of Health Research Center, Research Institute for prevention of Non-Communicable Diseases, Qazvin University of Medical Sciences, Shahid Bahonar Blvd, Qazvin 3419759811, Iran

5 Department of Nursing, School of Health and Welfare, Jönköping University, Jönköping, Sweden 